\title{
On the Dimensions of Automorphism Groups of Eight-Dimensional Ternary Fields, II
}

\author{
RICHARD BÖDI \\ Mathematisches Institut, Universität Tübingen, Auf der Morgenstelle 10, \\ D-72076 Tübingen, Germany \\ e-mail: mmisa01@convex.zdv.uni-tuebingen.de
}

(Received: 2 July 1993; revised version: 25 October 1993)

\begin{abstract}
Let $\mathcal{T}$ be an eight-dimensional, connected, locally compact ternary field and let $\Gamma$ denote a connected closed Lie subgroup of its automorphism group which is taken with the compact-open topology. It is proved that if the ternary fixed field $\mathcal{F}_{\Gamma}$ of $\Gamma$ is connected, then $\Gamma$ is either isomorphic to one of the compact Lie groups $G_{2}$ or $\mathrm{SU}_{3} \mathbb{C}$, or the (covering) dimension of $\Gamma$ is at most 7 .
\end{abstract}

Mathematics Subject Classification (1991): 51A35, 12J99, 22 E99.

This paper continues the study of automorphism groups $\Gamma$ of eight-dimensional locally compact connected ternary fields $\mathcal{T}$ in the sense of [14] and [3]. Throughout the paper we shall assume that $\Gamma$ is a closed subgroup of the (locally compact) automorphism group of $\mathcal{T}$. We shall work with the following definition of a topological ternary field, compare $[16,7.2]$.

(1) DEFINITION. A ternary field $\mathcal{T}=(T, 0,1, \tau)$ consists of a set $T$ containing the two distinct elements 0 and 1 and a ternary operation $\tau: T^{3} \rightarrow T:(s, x, t) \mapsto$ $\tau(s, x, t)$ which satisfies the following axioms:

(T1) $\tau(0, x, t)=\tau(x, 0, t)=t$ and $\tau(s, 1,0)=\tau(1, s, 0)=s$ for all $s, x, t \in T$.

(T2) For any elements $s, x, y \in T$ there exists a unique element $t=t(s, x, y)$ in $T$ such that $\tau(s, x, t)=y$.

(T3) For any elements $s_{1}, s_{2}, t_{1}, t_{2} \in T$ with $s_{1} \neq s_{2}$ there exist unique elements $x=x\left(s_{1}, t_{1}, s_{2}, t_{2}\right)$ and $s=s\left(s_{1}, t_{1}, s_{2}, t_{2}\right)$ in $T$ such that $\tau\left(s_{i}, x, t_{i}\right)=y$ and $\tau\left(s, s_{i}, t\right)=t_{i}$ holds for some $y, t \in T$ and $i=1,2$.

A ternary field $\mathcal{T}$ is called topological if $T$ is provided with a topology being neither discrete nor indiscrete such that the map $\tau$ and its inverse mappings $t, x$ and $s$ are continuous.

Using the terminology and notation of [3] the main results of the articles mentioned above are collected in the following

(2) THEOREM. The following conclusions hold:

(a) $\operatorname{dim} \Gamma \leq 14$. 
(b) If $\Gamma$ is a compact group, it is either isomorphic to one of the groups $G_{2}, \mathrm{SU}_{3} \mathbb{C}$ or $\mathrm{SO}_{4} \mathbb{R}$, or the inequalities $\operatorname{dim} \Gamma^{\prime} \leq 3, \operatorname{dim} \Gamma \leq 7$ and even $\operatorname{dim} \Gamma \leq 4$ if $\Gamma$ is a Lie group, hold.

(c) The group $\Gamma$ is compact or $\operatorname{dim} \Gamma \leq 11$.

(d) If the ternary fixed field $\mathcal{F}_{\Gamma}$ is connected, then $\Gamma$ is a compact group or $\operatorname{dim} \Gamma \leq 10$.

(e) A semi-simple, non-compact group $\Gamma$ is at most ten-dimensional.

(f) A commutative group $\Gamma$ is at most seven-dimensional.

We investigate the possible dimensions of the automorphism group $\Gamma$ in the case where $\Gamma$ is a Lie group and its ternary fixed field $\mathcal{F}_{\Gamma}$ is connected. The main result we shall prove is the alternative: 'Either the group $\Gamma$ is compact or its dimension is at most seven.' We shall start with a result on compact subgroups of $\Gamma$ in the case where $\Gamma$ is not compact.

(3) LEMMA. Let $\mathrm{K}$ be a maximal compact subgroup of $\Gamma$, where $\Gamma$ is a non-compact connected Lie group. Then $\mathrm{K}$ is isomorphic to either $\mathrm{Spin}_{3} \mathbb{R}$ or the circle group $\mathbb{T}$, or $\mathrm{K}=1$.

Proof. Since $\Gamma$ is not compact, it cannot contain commuting involutions by $[14,2.3]$. Thus the dimension of a maximal torus subgroup of $\Gamma$ is at most 1 . A compact connected Lie group of torus rank at most 1 is either isomorphic to one of the groups $\mathrm{Spin}_{3} \mathbb{R}, \mathrm{SO}_{3} \mathbb{R}$ or the circle group $\mathbb{T}$, or it is the trivial group. But the group $\mathrm{SO}_{3} \mathbb{R}$ does contain commuting involutions. Every maximal compact subgroup of a connnected Lie group is connected by the theorem of MalcevIwasawa [7]. Hence $K$ is isomorphic to one of the groups stated in the lemma.

Let $\mathcal{E}$ denote the smallest closed ternary subfield of $\mathcal{T}$. The next lemma shows that in proving the aforementioned alternative we may restrict our attention to the case where $\mathcal{F}_{\Gamma}=\mathcal{E}$. Note that this lemma does not presume $\Gamma$ to be a Lie group. The set $\sqrt{-1}:=\{x \in T \mid \tau(x, x, 1)=0\}$ is nonempty, compact and $\Gamma$-invariant by $[4, \mathrm{XI} .8 .11]$, see also $[3,2.2]$.

(4) LEMMA. If $\operatorname{dim} \mathcal{F}_{\Gamma}>0$ then one of the following statements holds:

(1) The group $\Gamma$ is compact.

(2) $\operatorname{dim} \Gamma \leq 7$.

(3) $\operatorname{dim} \mathcal{F}_{\Gamma}=1$ and $\operatorname{dim}\langle c\rangle=4$ for all $c \in \sqrt{-1}$.

Proof. By [9], we have $\operatorname{dim} \mathcal{F}_{\Gamma}=2^{m}$ with $0 \leq m \leq 3$. We may assume that $\operatorname{dim} \mathcal{F}_{\Gamma}=1$. Otherwise the assertion follows from $[3,3.1]$ and $[3,3.2]$. So we may choose an element $c \in \sqrt{-1} \backslash \mathcal{F}_{\Gamma}$ by $[3,2.2]$. If $\langle c\rangle=\mathcal{T}$ then $\operatorname{dim} \Gamma=\operatorname{dim} c^{\Gamma} \leq 7$ by $[3,2.3]$. Thus we may suppose that $\operatorname{dim}\langle c\rangle=2$ in view of statement $(3)$ of the lemma. Furthermore, we may assume by Proposition [3, 3.3] that the group $\Gamma$ is semi-simple. We shall show that $\Gamma$ is in fact a simple Lie group. For this purpose, we prove that the center $Z$ of $\Gamma$ is trivial. Suppose that $Z \neq 1$. Then either $\operatorname{dim} \mathcal{F}_{Z}=4$ or $\mathcal{F}_{Z}=\mathcal{F}_{\Gamma}$ holds by [2, (3.3)]. In the first case we conclude 
that the group $\Gamma$ is either compact or at most seven-dimensional by $[3,3.4]$ and $[14, \S 2,(2)]$. So let $\mathcal{F}_{Z}=\mathcal{F}_{\Gamma}$ and choose an element $\zeta \in Z \backslash 1$. As before there are two cases, namely $\operatorname{dim} \mathcal{F}_{\zeta}=4$ or $\mathcal{F}_{\zeta}=\mathcal{F}_{\Gamma}$, and as before we may restrict our attention to the second case. Thus we have $\mathcal{F}_{\zeta}=\mathcal{F}_{Z}=\mathcal{F}_{\Gamma}$ for every $\zeta \in Z \backslash$ l. If $\left\langle c^{Z}\right\rangle=\mathcal{T}$, then the stabilizer $\Gamma_{c}$ is trivial and $\operatorname{dim} \Gamma=\operatorname{dim} c^{\Gamma} \leq 7$ follows from $[3,2.3]$. If $\operatorname{dim}\left\langle c^{Z}\right\rangle=4$, then the group $\Gamma$ is compact by [14, 2.2], since we have the situation $\mathbb{C} \approx\langle c\rangle\left\langle\left\langle c^{\mathbb{Z}}\right\rangle\left\langle\mathcal{T}\right.\right.$. Thus the only remaining case is $\left\langle c^{\mathbb{Z}}\right\rangle=\langle c\rangle$. But then the center $Z$ acts effectively on the two-dimensional ternary subfield $\langle c\rangle$, since $\mathcal{F}_{\zeta}=\mathcal{F}_{\Gamma}$ for every $\zeta \in Z \backslash \mathbf{1}$. This implies that the center contains at most two elements, see [4, XI.9.3]. Since $\Gamma$ is finite-dimensional by Theorem (2), this implies that $\Gamma$ is a Lie group. Moreover, $\Gamma$ cannot contain a quasi-simple factor isomorphic to the universal covering of $\mathrm{SL}_{2} \mathbb{R}$, since $|Z| \leq 2$ holds. In particular, the group $\Gamma$ must be quasi-simple, because otherwise it would contain a two-dimensional torus subgroup and thus would be compact by $[14,2.3]$. Finally, if $|Z|=2$ then the group $\Gamma$ contains a central involution $\omega$ which has a Baer ternary fixed field. But this is impossible, since for a quasi-simple group of dimension at least 6 we have $\mathcal{F}_{\Gamma}=\mathcal{F}_{\omega}$, see $[1,(2.7)]$. Thus we have shown that $\Gamma$ is a simple Lie group. Now, the classification of quasi-simple Lie groups shows that a simple Lie group of dimension at least 8 always contains commuting involutions. This implies that either the group $\Gamma$ is compact by $[14,2.3]$ or $\operatorname{dim} \Gamma \leq 7$ holds.

The preceding lemma shows that for proving the inequality dim $\Gamma \leq 7$ in the case of a non-compact Lie group $\Gamma$ we may assume that $\operatorname{dim} \mathcal{F}_{\Gamma}=1$ and $\operatorname{dim}\langle c\rangle=4$ for every $c \in \mathcal{T} \backslash \mathcal{E}$. These assumptions are always taken for granted for the rest of this paper.

We introduce the following notations. For any element $c \in \mathcal{T}$ set $\mathcal{H}_{c}:=\langle c\rangle$. The set $\mathcal{V}:=\{c \in \mathcal{T} \mid \operatorname{dim}\langle c\rangle=4\}$ is regarded as a topological subspace of $\mathcal{T}$ taken with the relative topology $\tau_{\mathcal{V}}$. Set $\mathcal{B}:=\left\{\mathcal{H}_{c} \mid c \in \mathcal{V}\right\}$ and let $\mathcal{H}^{*}:=\mathcal{H} \cap \mathcal{V}$ for a ternary subfield $\mathcal{H} \leq \mathcal{T}$. Finally, the relative topology of the subset $\sqrt{-1}$ in $\mathcal{T}$ is denoted by $\tau_{\sqrt{-1}}$. We use the abbreviation $\mathrm{Cs}$ to denote a centralizer. By the symbol $\approx$ we denote a homeomorphy.

The main idea for estimating the upper bound for the dimension of $\Gamma$ is the detailed study of the action of $\Gamma$ on the set $\mathcal{B}$. We shall show that $\mathcal{B}$ can be topologized in such a way that $\mathcal{B}$ becomes a compact analytical manifold which is homeomorphic to some quotient space $\mathcal{M}$ of $\Gamma$. Thus, the group $\Gamma$ acts on $\mathcal{B}$ as a topological transformation group. This fact will provide additional information about the structure of the group $\Gamma$. The main tool for this procedure is the following rather technical lemma.

(5) LEMMA. Let $\Gamma$ be a non-compact Lie group. Suppose that the following conditions are satisfied:

(I) $\sqrt{-1} \subseteq \mathcal{V}$,

(II) for every $c \in \mathcal{V}$ the stabilizer $\Gamma_{c}$ contains a unique involution, 
(III) there is an involution $\omega \in \Gamma$ such that $\operatorname{dim} \Gamma / \mathrm{Cs}(\omega) \geq 4$, (IV) $\Gamma$ acts transitively on $\mathcal{B}$.

Then the quotient space $\mathcal{M}:=\Gamma / \operatorname{Cs}(\omega)$ is a compact four-dimensional analytical manifold with respect to the quotient topology $\tau$. Moreover, $\operatorname{Cs}(\omega)=\Gamma_{\left[\mathcal{F}_{\omega}\right]}$ holds.

Proof. From condition (I) we can easily deduce the following assertion (V) If $\mathcal{H} \in \mathcal{B}$ and $\mathcal{U}<\mathcal{H}$, then $\mathcal{U}=\mathcal{E}$.

To prove this, let $\mathcal{U}<\mathcal{H} \in \mathcal{B}$. If $\mathcal{U} \neq \mathcal{E}$, then $\operatorname{dim} \mathcal{U}=2$ and $\mathcal{U} \cap \sqrt{-1} \neq \emptyset$ because of $\mathcal{E} \approx \mathbb{R}$, see [4, XI.8.11]. But for each element $c \in \mathcal{U} \cap \sqrt{-1}$ we have $\langle c\rangle=\mathcal{U}$ which contradicts condition (I).

If $\mathcal{H}$ is an arbitrary Baer ternary subfield and if $c \in \mathcal{H}^{*}$, then $\mathcal{H}=\langle c\rangle$, otherwise we would have a tower of ternary subfields $\mathbb{C} \approx\langle c\rangle\langle\mathcal{H}\langle\mathcal{T}$ and the group would be compact by [14]. Hence we have

(VI) Every element $c \in \mathcal{H}^{*}$ of a Baer ternary subfield $\mathcal{H}$ generates $\mathcal{H}$ and $\mathcal{H}^{*}=\mathcal{H} \backslash \mathcal{E}$.

The proof of the lemma is divided into five steps. In the first step we shall define four topologies $\tau_{1}, \ldots, \tau_{4}$ on the set $\mathcal{B}$, where each of these topologies has certain properties, which can easily be verified. In particular, it will be obvious that $\left(\mathcal{B}, \tau_{4}\right)$ is homeomorphic to $\mathcal{M}$. In steps (2) and (3) we shall verify that the topologies $\tau_{1}, \tau_{2}$, and $\tau_{4}$ coincide with the quotient topology $\tau$ on $\mathcal{M}$. This yields three different descriptions of the topology $\tau$ on $\mathcal{M}$. Step (3) also yields the assertion that $\mathcal{M}$ is four-dimensional. In the last step we shall prove that the (Hausdorff) compact topology $\tau_{3}$ is finer than the Hausdorff topology $\tau$. In particular, this shows the compactness of $\left(\mathcal{B}, \tau_{4}\right) \approx \mathcal{M}$.

STEP (1). Definition of the topologies $\tau_{1}, \ldots, \tau_{4}$.

The topology $\tau_{1}$.

Let $\tau_{1}$ be the quotient topology of $\left(\mathcal{V}, \tau_{\mathcal{V}}\right)$ with respect to the map

$$
\varphi_{1}: \mathcal{V} \rightarrow \mathcal{B}: c \mapsto \mathcal{H}_{c} .
$$

Assertion (V) implies that $\varphi_{1}^{\leftarrow}(\mathcal{O})=\bigcup_{\mathcal{H} \in \mathcal{O}} \mathcal{H}^{*}$ for every subset $\mathcal{O} \subseteq \mathcal{B}$. Hence we have

$$
\mathcal{O} \in \tau_{1} \text { iff } \bigcup_{\mathcal{H} \in \mathcal{O}} \mathcal{H}^{*} \in \tau_{\mathcal{V}} .
$$

Since $\Gamma$ induces a topological transformation group on $\mathcal{V}$ and because $\varphi_{1}$ is continuous by construction, $\Gamma$ is a topological transformation group on $\left(\mathcal{B}, \tau_{1}\right)$. Thus, the topological space $\left(\mathcal{B}, \tau_{1}\right)$ is homogeneous by condition (IV).

The topology $\tau_{2}$. It is easily seen that by

$$
\mathcal{O} \in \tau_{2}: \Longleftrightarrow \exists U \in \tau_{\mathcal{V}}:(\mathcal{H} \in \mathcal{O} \Longleftrightarrow \mathcal{H} \cap U \neq \emptyset)
$$

a topology $\tau_{2}$ on $\mathcal{B}$ is defined. A set $\mathcal{O}$ is open with respect to $\tau_{2}$ iff it can be written in the form $\{\mathcal{H} \in \mathcal{B} \mid \mathcal{H} \cap U \neq \emptyset\}$ for some open subset $U \in \tau_{\mathcal{V}}$. 
The topology $\tau_{3}$. Consider the mapping

$$
\varphi_{3}: \sqrt{-1} \rightarrow \mathcal{B}: c \mapsto \mathcal{H}_{c} .
$$

For every element $c \in \sqrt{-1}$ there is some Baer ternary subfield $\mathcal{H} \in \mathcal{B}$ with $c \in \mathcal{H}$ (namely $\mathcal{H}=\mathcal{H}_{c}$ ) by condition (I). Since we have $\mathcal{E} \cap \sqrt{-1}=\emptyset$ (see $[3,2.2]$ ), condition $(\mathrm{V})$ states that the Baer ternary subfield $\mathcal{H}_{c}$ is uniquely determined by the element $c$. Thus the map $\varphi_{3}$ is well defined. Since every ternary subfield $\mathcal{H} \in \mathcal{B}$ meets the set $\sqrt{-1}\left[4\right.$, XI.8.11], the map $\varphi_{3}$ is surjective. Hence we may define $\tau_{3}$ to be the final topology of $\left(\sqrt{-1}, \tau_{\sqrt{-1}}\right)$ with respect to $\varphi_{3}$. Thus we have

$$
\mathcal{O} \in \tau_{3} \Longleftrightarrow \varphi_{3}^{\leftarrow}(\mathcal{O})=\bigcup_{\mathcal{H} \in \mathcal{O}}(\mathcal{H} \cap \sqrt{-1}) \in \tau_{\sqrt{-1}}
$$

Being a continuous image of the compact set $\sqrt{-1}$, the space $\left(\mathcal{B}, \tau_{3}\right)$ is compact (not, necessarily Hausdorff). As in the case of the definition of the topology $\tau_{1}$, the group $\Gamma$ is a topological transformation group on $\left(\mathcal{B}, \tau_{3}\right)$ and thus by condition (IV) the space $\left(\mathcal{B}, \tau_{3}\right)$ is homogeneous.

The topology $\tau_{4}$. Let $\omega \in \Gamma$ be an involution which satisfies condition (III). Consider the mapping

$$
\varphi_{4}: \Gamma / \operatorname{Cs}(\omega) \rightarrow \mathcal{B}:(\operatorname{Cs}(\omega)) \gamma \mapsto \mathcal{F}_{\omega}^{\gamma} .
$$

In order to verify that the map $\varphi_{4}$ is well defined and injective we prove

$$
\text { An automorphism } \gamma \in \Gamma \text { leaves } \mathcal{F}_{\omega} \text { invariant iff } \gamma \in \mathrm{Cs}(\omega)
$$

Obviously, every element $\gamma \in \operatorname{Cs}(\omega)$ leaves $\mathcal{F}_{\omega}$ invariant. Conversely, let $\gamma \in \Gamma$ with $\mathcal{F}_{\omega}^{\gamma}=\mathcal{F}_{\omega}$. By (VI) there is an element $c \in \mathcal{F}_{\omega}$ with $\mathcal{F}_{\omega}=\langle c\rangle$. Then, by (VI), we have $\langle c\rangle=\mathcal{F}_{\omega}=\mathcal{F}_{\omega}^{\gamma}=\left\langle c^{\gamma}\right\rangle$ and this implies that $\Gamma_{c}=\Gamma_{c \gamma}$. If $\omega \gamma \neq \gamma \omega$, then $\omega^{\prime}=\omega^{\gamma}$ would be an involution with ternary fixed field $\mathcal{F}_{\omega^{\prime}} \neq \mathcal{F}_{\omega}$, since the stabilizer $\Gamma_{c}$ contains a single involution by condition (II). As before, there is some element $a \in \mathcal{T}$ which generates $\mathcal{F}_{\omega^{\prime}}$. Now we have $a^{\gamma^{-1} \omega}=a^{\gamma^{-1}}$, because the element $a$ is fixed by the involution $\omega^{\prime}$. Thus the element $a^{\gamma^{-1}}$ is contained in $\mathcal{F}_{\omega}$ and we infer that $a=\left(a^{\gamma^{-1}}\right)^{\gamma} \in \mathcal{F}_{\omega}^{\gamma}=\mathcal{F}_{\omega}$. Consequently, we have $a \in \mathcal{F}_{\omega} \cap \mathcal{F}_{\omega^{\prime}}$. On the other hand $\mathcal{F}_{\omega} \cap \mathcal{F}_{\omega^{\prime}}=\mathcal{E}$ holds by condition (V). But this is impossible, since the element $a$ generates $\mathcal{F}_{\omega^{\prime}} \neq \mathcal{E}$. This contradiction shows that the automorphisms $\omega$ and $\gamma$ commute and $(*)$ is verified.

In particular, assertion $(*)$ proves the equation $\operatorname{Cs}(\omega)=\Gamma_{\left[\mathcal{F}_{w]}\right]}$. Since the map $\varphi_{4}$ is a surjection by condition (IV), we may define $\tau_{4}$ to be the topology on $\mathcal{B}$ with respect to which $\varphi_{4}$ becomes a homeomorphism between $\Gamma / \operatorname{Cs}(\omega)$ and $\left(\mathcal{B}, \tau_{4}\right)$.

STEP (2). The topologies $\tau_{1}$ and $\tau_{2}$ coincide.

Let $\mathcal{O} \in \tau_{1}$. Then we have $\mathcal{U}:=\bigcup_{\mathcal{H} \in \mathcal{O}} \mathcal{H}^{*} \in \tau_{\mathcal{V}}$ by definition of the topology $\tau_{1}$. Condition (V) implies that this union is a disjoint union. Hence a Baer ternary 
subfield $\mathcal{H}$ is contained in $\mathcal{O}$ if and only if the intersection $\mathcal{H} \cap \mathcal{U}$ is nonempty, and we infer that $\mathcal{O} \in \tau_{2}$. Thus the topology $\tau_{2}$ is finer than the topology $\tau_{1}$. To prove the converse, select an element $\mathcal{O} \in \tau_{2}$. By definition of $\tau_{2}$ there is a set $\mathcal{U} \in \tau_{\mathcal{V}}$ such that $\mathcal{O}=\{\mathcal{H} \in \mathcal{B} \mid \mathcal{H} \cap \mathcal{U} \neq \emptyset\}$. Suppose that the set $\mathcal{O}$ is not open with respect to the topology $\tau_{1}$. Then $\mathcal{X}:=\bigcup_{\mathcal{H} \in \mathcal{O}} \mathcal{H}^{*} \subseteq \mathcal{V}$ is not open with respect to the topology $\tau_{\mathcal{V}}$. Thus we may choose an element $c \in \mathcal{X} \cap \partial \mathcal{X}$. Set $\mathcal{Y}:=\mathcal{V} \backslash \mathcal{X}$. Since the tenary field $\mathcal{T}$ has a countable base (see [4, XI.1.2, XI.2.2]), there is a sequence $\left(c_{n}\right)_{n \in \mathbb{N}}$ in $\mathcal{Y}$ converging to $c$ and $\operatorname{so} \operatorname{dim}\left\langle c_{n}\right\rangle=\operatorname{dim} \mathcal{H}_{c_{n}}=4$ holds for every $n \in \mathbb{N}$. Consider the set

$$
\mathcal{A}:=\left\{a \in \mathcal{H}_{c} \mid\left[\forall n \in \mathbb{N} \exists a_{n} \in \mathcal{T}: a_{n} \in \mathcal{H}_{c_{n}}\right] \text { and } \lim _{n \rightarrow \infty} a_{n}=a\right\} .
$$

Then $\mathcal{A}$ is a (not necessarily closed) ternary subfield of $\mathcal{H}_{c}$. To prove this, choose elements $s_{n}, t_{n}, u_{n}, v_{n} \in \mathcal{H}_{c_{n}}$ with

$$
\lim _{n \rightarrow \infty} s_{n}=s, \lim _{n \rightarrow \infty} t_{n}=t, \lim _{n \rightarrow \infty} u_{n}=u, \lim _{n \rightarrow \infty} v_{n}=v
$$

where $s, t, u, v \in \mathcal{H}_{c}$. Then we have

$$
\tau(s, u, t)=\tau\left(\lim _{n \rightarrow \infty} s_{n}, \lim _{n \rightarrow \infty} u_{n}, \lim _{n \rightarrow \infty} t_{n}\right)=\lim _{n \rightarrow \infty} \tau\left(s_{n}, u_{n}, t_{n}\right)
$$

which implies that $\tau(s, u, t) \in \mathcal{A}$. Similar, we are able to deduce from

$$
t(s, u, v)=t\left(\lim _{n \rightarrow \infty} s_{n}, \lim _{n \rightarrow \infty} u_{n}, \lim _{n \rightarrow \infty} v_{n}\right)=\lim _{n \rightarrow \infty} t\left(s_{n}, u_{n}, v_{n}\right)
$$

the relation $t(s, u, v) \in \mathcal{A}$. The verification of the last axiom (T3) of a ternary field is done in much the same way; given two distinct elements $s, s^{\prime} \in \mathcal{A}$, we only have to choose sequences $\left(s_{n}\right)$ and $\left(s_{n}^{\prime}\right)$ with $s_{n}, s_{n}^{\prime} \in \mathcal{H}_{c_{n}}$ which converge to $s$ and $s^{\prime}$, respectively, such that $s_{n} \neq s_{n}^{\prime}$ holds for every $n \in \mathbb{N}$. This can be achieved by passing to appropriate subsequences.

Since $c \in \mathcal{A}$, the closure $\langle\mathcal{A}\rangle$ of $\mathcal{A}$ in $\mathcal{T}$ is just $\mathcal{H}_{c}$, i.e. $\mathcal{A}$ is dense in $\mathcal{H}_{c}$. Because the intersection $\mathcal{U} \cap \mathcal{H}_{c}$ is open in $\mathcal{H}_{c}$, there is an element $d \in \mathcal{A} \cap \mathcal{U}$. By the very definition of $\mathcal{A}$ there is a sequence $\left(d_{n}\right)_{n \in \mathbb{N}}$ in $\mathcal{V}$ with $d_{n} \in \mathcal{H}_{c_{n}}$ and $\lim _{n \rightarrow \infty} d_{n}=d$. Thus there is an integer $m \in \mathbb{N}$ with $d_{m} \in \mathcal{U}$ and we infer that $\mathcal{H}_{d_{m}} \in \mathcal{O}$. In particular, we have $d_{m} \in \mathcal{X}$. But this is impossible by the relations $\left\langle d_{m}\right\rangle=\mathcal{H}_{c_{m}}$ and $\mathcal{H}_{c_{m}} \cap \mathcal{X}=\emptyset$. Consequently, this contradiction shows that the set $\mathcal{O}$ is open with respect to the topology $\tau_{1}$. Hence the topologies $\tau_{1}$ and $\tau_{2}$ coincide. Moreover, this shows that $\varphi_{1}$ is an open map (use [8, Th. 10, p. 97], e.g.).

STEP (3). The topologies $\tau_{2}$ and $\tau_{4}$ coincide.

Let $\omega$ be an involution in the sense of (III) and consider the following diagram 


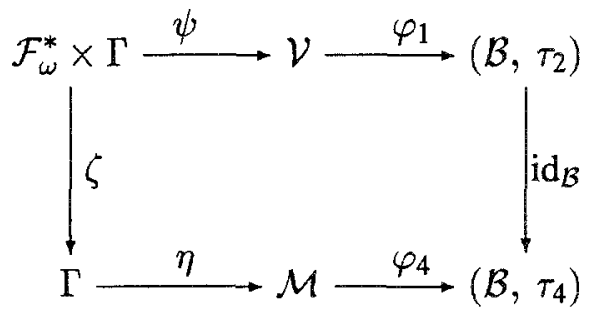

where $\psi=\left((c, \gamma) \mapsto c^{\gamma}\right)$ denotes the action of $\Gamma$ on $\mathcal{F}_{\Gamma}^{*}, \zeta$ is the projection onto the second factor, and $\eta$ is the canonical epimorphism from $\Gamma$ onto $\mathcal{M}=\Gamma / \operatorname{Cs}(\omega)$. This diagram is commutative, since for all pairs $(c, \gamma) \in \mathcal{F}_{\omega}^{*} \times \Gamma$ we have $\mathcal{H}_{c}=\mathcal{F}_{\omega}$ which implies that

$$
(c, \gamma) \psi \varphi_{1} \operatorname{id}_{\mathcal{B}}=\left(c^{\gamma}\right) \varphi_{1}=\mathcal{H}_{c^{\gamma}}=\mathcal{H}_{c}^{\gamma}=\mathcal{F}_{\omega}^{\gamma}
$$

and

$$
(c, \gamma) \zeta \eta \varphi_{4}=\gamma \eta \varphi_{4}=((\operatorname{Cs}(\omega)) \gamma) \varphi_{4}=\mathcal{F}_{\omega}^{\gamma}
$$

hold. By construction,

(1) the mappings $\varphi_{1}, \psi, \zeta, \eta$, and $\varphi_{4}$ are continuous, and

(2) the mappings $\zeta, \eta, \varphi_{4}$, and $\varphi_{1}$ (note that $\tau_{1}=\tau_{2}$ ) are open.

Note that the set $\mathcal{F}_{\omega}^{*} \times \Gamma$ is taken with the product topology.

The commutativity of the diagram implies that the identity map $\operatorname{id}_{\mathcal{B}}$ is open by properties (1) and (2), i.e. the topology $\tau_{4}$ is finer than $\tau_{2}$. To verify that the topology $\tau_{4}$ is coarser than the topology $\tau_{2}$, we have to do some further preparation. Note first that the product $\mathcal{F}_{\omega}^{*} \times \Gamma$ is locally compact with respect to the product topology, since $\Gamma$ and $\mathcal{F}_{\omega}$ are locally compact and $\mathcal{F}_{\omega}^{*}=\mathcal{F}_{\omega} \backslash \mathcal{E}$ is open in $\mathcal{F}_{\omega}$. Thus there is a nonempty, open, relative compact set $\mathcal{W} \times \Omega$ in $\mathcal{F}_{\omega}^{*} \times \Gamma$. Set $C:=\overline{\mathcal{W}} \times \bar{\Omega}$. Because $C$ is compact, the restriction $\psi^{\prime}$ of $\psi$ to the set $C$ is a closed mapping. Moreover, the set $\mathcal{F}_{\omega}^{*} \times \Gamma$ has a countable base, since $\Gamma$ is a Lie group. So we may apply $[13,9.2 .6]$ to $\psi^{\prime}$ and by $[2,3.1]$ we obtain that

$$
\operatorname{dim}\left(\mathcal{F}_{\omega} \times \Gamma\right)=\operatorname{dim}\left(\mathcal{F}_{\omega}^{*} \times \Gamma\right)=\operatorname{dim} C \leq \operatorname{dim} \psi^{\prime}+\operatorname{dim} C \psi^{\prime},
$$

where $\operatorname{dim} \psi^{\prime}=\sup \left\{\operatorname{dim}\left(\psi^{\prime \leftarrow}(y)\right) \mid y \in \mathcal{V}\right\}$. By [2, (3.1)] this implies that

$$
\operatorname{dim} C \psi^{\prime} \geq \operatorname{dim}\left(\mathcal{F}_{\omega} \times \Gamma\right)-\operatorname{dim} \psi^{\prime}=4+\operatorname{dim} \Gamma-\operatorname{dim} \psi^{\prime} .
$$

For every element $y \in \mathcal{V}$ we have

$$
S_{y}:=\psi^{\prime \leftarrow}(y)=\left\{(c, \gamma) \in C \mid c^{\gamma}=y\right\} .
$$


We shall show that the restriction $\zeta^{\prime}:=\left.\zeta\right|_{S_{y}}: S_{y} \rightarrow S_{y} \zeta \subseteq \Gamma$ is a homeomorphism. Being a projection, the $\operatorname{map} \zeta$ is continuous and open. So we only have to prove that $\zeta^{\prime}$ is injective. For this, choose elements $\left(c_{1}, \gamma_{1}\right),\left(c_{2}, \gamma_{2}\right) \in S_{y}$ with $\left(c_{1}, \gamma_{1}\right) \zeta^{\prime}=$ $\left(c_{2}, \gamma_{2}\right) \zeta^{\prime}=\delta$. Then $\gamma_{1}=\gamma_{2}=\delta$ and $c_{1}^{\delta}=c_{2}^{\delta}$. Since $\delta$ is an automorphism of the ternary field $\mathcal{T}$, this implies that $c_{1}=c_{2}$, i.e., $\zeta^{\prime}$ is an injection. Thus for every element $y \in C \psi^{\prime} \subseteq \mathcal{V}$ we have

$$
\operatorname{dim} \psi^{\prime \leftarrow}(y)=\operatorname{dim} S_{y}=\operatorname{dim} S_{y} \zeta^{\prime} .
$$

Next, we want to prove that

$$
\operatorname{dim} S_{y} \zeta^{\prime} \leq \operatorname{dim} \operatorname{Cs}(\omega)
$$

In order to derive this inequality, let $\gamma \in S_{y} \zeta^{\prime}$. Then there is some element $c \in$ $\overline{\mathcal{W}} \subseteq \mathcal{F}_{\omega}$ such that $c^{\gamma}=y$. Hence we have by (VI) that

$$
\mathcal{F}_{\omega}^{\gamma}=\langle c\rangle^{\gamma}=\left\langle c^{\gamma}\right\rangle=\langle y\rangle
$$

for every automorphism $\gamma \in S_{y} \zeta^{\prime}$. Since by condition (IV) the group $\Gamma$ acts transitively on the set $\mathcal{B}$, there is some $\delta \in \Gamma$ such that

$$
\mathcal{F}_{\omega}^{\gamma \delta}=\mathcal{F}_{\omega}
$$

holds for all $\gamma \in S_{y} \zeta^{\prime}$. By assertion (*) of Step (1) this implies the inclusion

$$
\left(S_{y} \zeta^{\prime}\right) \delta \subseteq \operatorname{Cs}(\omega)
$$

Because $S_{y} \approx S_{y} \zeta^{\prime} \approx S_{y} \zeta^{\prime} \delta$ is compact and thus is closed by the continuity of the map $\psi^{\prime}$, we may apply the sum theorem (see [13, 3.2.5], e.g.) and obtain the desired inequality

$$
\operatorname{dim} S_{y} \zeta^{\prime}=\operatorname{dim} S_{y} \zeta^{\prime} \delta \leq \operatorname{dim} \operatorname{Cs}(\omega) .
$$

Since this holds for every element $y$ in $C \psi^{\prime} \subseteq \mathcal{V}$, we infer that

$$
\operatorname{dim} \psi^{\prime}=\sup \left\{\operatorname{dim}\left(\psi^{\prime \leftarrow}(y)\right) \mid y \in \mathcal{V}\right\} \leq \operatorname{dim} \operatorname{Cs}(\omega) .
$$

Substituting this result in $(\diamond)$ we obtain by condition (III) that

$$
\operatorname{dim} C \psi^{\prime} \geq \operatorname{dim} \Gamma-\operatorname{dim} \operatorname{Cs}(\omega)+4=\operatorname{dim} \mathcal{M}+4 \geq 8 .
$$

Because $C \psi=C \psi^{\prime} \subseteq \mathcal{T}$ is compact, we have $\operatorname{dim} C \psi=8$ by the sum theorem and thus

$$
\operatorname{dim} \mathcal{M}=\operatorname{dim} \Gamma-\operatorname{dim} \operatorname{Cs}(\omega)=4 .
$$


Moreover, $\operatorname{dim} C \psi=8$ implies that the set $C \psi$ contains inner points (see [1,(3.1)]).

Now we are able to prove that the topology $\tau_{2}=\tau_{1}$ is finer than the topology $\tau_{4}$. Since the spaces $\left(\mathcal{B}, \tau_{1}\right)$ and $\left(\mathcal{B}, \tau_{4}\right)$ are homogeneous, it suffices to consider neighborhoods of $\mathcal{F}_{\omega}$. So let $\mathcal{U} \in \tau_{4}$ be a neighborhood of $\mathcal{F}_{\omega}$. Because the map $\zeta \eta \varphi_{4}$ is continuous and $\mathcal{F}_{\omega}^{*} \times \Gamma$ is locally compact, there exists a relatively compact and open set $\mathcal{W} \times \Omega \subset \mathcal{F}_{\omega}^{*} \times \Gamma$ with $\mathbb{1} \in \Omega$ satisfying

$$
(\mathcal{W} \times \Omega) \zeta \eta \varphi_{4} \subseteq \mathcal{U}
$$

Furthermore, let $\Theta$ be a relatively compact open neighborhood of $\mathbb{1} \in \Gamma$ such that $\Theta \Theta^{-1}$ is contained in $\Omega$. As proved above, the set $\mathcal{O}:=((\mathcal{W} \times \Theta) \psi)^{\circ}=\left(\mathcal{W}^{\Theta}\right)^{\circ}$ is nonempty. Hence we may choose an element $(c, \gamma) \in \mathcal{W} \times \Theta$ with $c^{\gamma} \in \mathcal{O}$. Then we have

$$
c=c^{\gamma \gamma^{-1}} \in \mathcal{O}^{\gamma^{-1}} \subseteq\left(\left(\mathcal{W}^{\Theta}\right)^{\circ}\right)^{\Theta^{-1}}=\left(\mathcal{W}^{\Theta \Theta^{-1}}\right)^{\circ} \subseteq\left(\mathcal{W}^{\Omega}\right)^{\circ}=: \mathcal{O}^{\prime} .
$$

By definition of the topology $\tau_{1}$ this means that $\mathcal{F}_{\omega}=\langle c\rangle \in \mathcal{O}^{\prime} \varphi_{1}$. Hence

$$
\mathcal{O}^{\prime} \varphi_{1} \subseteq(\mathcal{W} \times \Omega) \psi \varphi_{1}=(\mathcal{W} \times \Omega) \zeta \eta \varphi_{4} \subseteq \mathcal{U}
$$

follows. The $\operatorname{set} \mathcal{O}^{\prime} \varphi_{1} \subseteq \mathcal{U}$ is an open neighborhood of $\mathcal{F}_{\omega}$ with respect to $\left(\mathcal{B}, \tau_{1}\right)=$ $\left(\mathcal{B}, \tau_{2}\right)$, because $\mathcal{O}^{\prime}$ is an open set and $\varphi_{1}$ is an open map. Thus we have verified that the topology $\tau_{2}$ is finer than $\tau_{4}$.

STEP (4). The topology $\tau_{3}$ is finer than the topology $\tau_{1}$.

Let $\mathcal{O} \in \tau_{1}$. By definition of the topology $\tau_{1}$ we have $\bigcup_{\mathcal{H} \in \mathcal{O}} \mathcal{H}^{*} \in \tau_{\mathcal{V}}$. By condition (I) the relation $\tau_{\sqrt{-1}}=\left\{\mathcal{U} \cap \sqrt{-1} \mid \mathcal{U} \in \tau_{\mathcal{V}}\right\}$ holds. Combining both results, we obtain that

$$
\left(\bigcup_{\mathcal{H} \in \mathcal{O}} \mathcal{H}^{*}\right) \cap \sqrt{-1}=\bigcup_{\mathcal{H} \in \mathcal{O}}\left(\mathcal{H}^{*} \cap \sqrt{-1}\right) \in \tau_{\sqrt{-1}} .
$$

Thus, the set $\mathcal{O}$ is open with respect to $\tau_{3}$, which proves that $\tau_{3}$ is finer than $\tau_{1}$.

$\operatorname{STEP}(5) .(\mathcal{M}, \tau)$ is a compact analytical manifold.

By steps (2), (3) and (4), we have the relations

$$
\tau_{4}=\tau_{2}=\tau_{1} \prec \tau_{3} .
$$

Being a continuous image of the compact space $\left(\sqrt{-1}, \tau_{\sqrt{-1}}\right)$, the space $\left(\mathcal{B}, \tau_{3}\right)$ is quasi-compact. In particular, $\left(\mathcal{B}, \tau_{4}\right) \approx \mathcal{M}$ is quasi-compact because of $\tau_{4}=$ $\tau_{1} \prec \tau_{3}$. But since $\Gamma$ is a Lie group, the quotient $\mathcal{M}=\Gamma / \operatorname{Cs}(\omega)$ is an analytical manifold. Thus, all claims of the lemma are verified.

If $\Gamma$ is a non-compact Lie group of dimension at least 8, condition (I) of Lemma (5) is satisfied by Theorem (4). The next lemma shows that in this case conditions (II) 
and (IV) are also satisfied.

(6) LEMMA. Let $\Gamma$ be a non-compact Lie group with $\operatorname{dim} \Gamma \geq 8$. Then $\Gamma$ acts transitively on the set $\mathcal{B}$ of all Baer ternary subfields of $\mathcal{T}$. Moreover, for every element $c \in \mathcal{V}$ the stabilizer $\Gamma_{c}$ is isomorphic either to $\operatorname{Spin}_{3} \mathbb{R}$ or to a circle group.

Proof. Choose an element $c \in \mathcal{V}$. Since the ternary subfield $\mathcal{H}_{c}$ is fourdimensional, the stabilizer $\Gamma_{c}$ is a compact Lie group [14, (2.3)]. According to Lemma (3), the subgroup $\Gamma_{c}^{1}$ is isomorphic to one of the groups $1, \mathbb{T}$ (circle group), or $\operatorname{Spin}_{3} \mathbb{R}$. The ternary subfield $\mathcal{H}_{c}$ is generated by any element $d$ of $\mathcal{H}_{c} \backslash \mathcal{E}$ (cf. (VI) in the proof of Lemma (5)). This implies that $\Gamma_{c}=\Gamma_{d}$ for every $d \in \mathcal{H}_{c} \backslash \mathcal{E}$. Because of $\mathcal{H}_{c} \cap \sqrt{-1} \neq \emptyset$ we may choose the element $c$ in $\sqrt{-1}$. Applying [3, 2.3] we obtain that $\operatorname{dim} c^{\Gamma} \leq 7$. Since $\operatorname{dim} \Gamma \geq 8$, this implies that $\operatorname{dim} \Gamma_{c} \geq 1$ and thus $\Gamma_{c}^{1}$ is isomorphic to either the circle group $\mathbb{T}$ or to $\operatorname{Spin}_{3} \mathbb{R}$.

Now let $\mathrm{K}$ be a maximal compact subgroup of $\Gamma$ such that $\Gamma_{c} \leq \mathrm{K}$. Again, by Lemma (3), we know that $K$ is a subgroup of $\operatorname{Spin}_{3} \mathbb{R}$. Thus, if $\operatorname{dim} \Gamma_{c}=\operatorname{dim} K$ then $\Gamma_{c}=\mathrm{K}$. If, on the other hand, the identity component $\Gamma_{c}^{1}$ of the stabilizer $\Gamma_{c}$ is a circle group and $\mathrm{K} \cong \mathrm{Spin}_{3} \mathbb{R}$, we consider the unique involution $\omega$ of $K$. Therefore $\omega \in \Gamma_{c}$ and $\mathrm{K}_{c}=\mathcal{F}_{\omega}=\mathcal{H}_{c}$. Hence the compact group $\mathrm{K}$ acts on $\mathcal{H}_{c}=\mathcal{F}_{\omega}$ and the stabilizer $\Gamma_{c}^{1}$ is contained in the kernel of this action. Consequently, the group $\mathrm{K}$ acts trivially on $\mathcal{H}_{c}$, because $\mathrm{K}$ is quasi-simple. But this contradicts the assumption that $\Gamma_{c}^{1}<\mathrm{K}$. In particular, the stabilizer $\Gamma_{c}$ is always a maximal compact subgroup of $\Gamma$ and thus is connected.

For a verification of the transitivity statement let $\mathcal{H}_{c}, \mathcal{H}_{d} \in \mathcal{B}$. By what we have proved above, the stabilizers $\Gamma_{c}$ and $\Gamma_{d}$ are maximal compact subgroups of $\Gamma$ and thus are conjugated in $\Gamma$. So there is an element $\alpha \in \Gamma$ such that $\Gamma_{c}^{\alpha}=\Gamma_{d}$. From $\Gamma_{c}^{\alpha}=\Gamma_{c^{\alpha}}=\Gamma_{d}$ we infer that $\left\langle c^{\alpha}\right\rangle=\langle d\rangle$ which implies that $\mathcal{H}_{c}^{\alpha}=\langle c\rangle^{\alpha}=\left\langle c^{\alpha}\right\rangle=\langle d\rangle=\mathcal{H}_{d}$. This proves that the group $\Gamma$ acts transitively on the set $\mathcal{B}$.

Using the preceding lemma we are able to prove:

(7) THEOREM. If $\Gamma$ is a non-compact, semi-simple Lie group, then $\operatorname{dim} \Gamma \leq 6$.

Proof. By [3, 3.5] we have $\operatorname{dim} \Gamma \leq 10$. First let $\operatorname{dim} \Gamma \geq 8$. A maximal compact subgroup $K$ of $\Gamma$ must be isomorphic to a closed subgroup of $\operatorname{Spin}_{3} \mathbb{R}$ by Lemma (3). We shall study the dimensions 8, 9, and 10 separately.

CASE $1 . \operatorname{dim} \Gamma=10$. A 10-dimensional semi-simple Lie group $\Gamma$ is quasisimple by the classification of quasi-simple Lie groups, and $\Gamma$ is locally isomorphic to one of the groups $\mathrm{SO}_{5, i} \mathbb{R}$ with $0 \leq i \leq 2$, see [17], for instance. By Lemma (3), this implies that $\mathrm{K} \cong \mathrm{Spin}_{3} \mathbb{R}$ and $\Gamma$ must be isomorphic to the universal covering of the group $\mathrm{SO}_{5,2} \mathbb{R}$. But this group contains a central involution $\omega$ and thus $\Gamma$ acts on the Baer ternary fixed field $\mathcal{F}_{\omega}$. Since $\Gamma$ is a quasi-simple group of dimension greater than 4 , the group $\Gamma$ must fix the Baer ternary subfield $\mathcal{F}_{\omega}$ pointwise, see [1] and $[2,(3.3)]$. This, however, is a contradiction to $[3,3.1]$. 
CASE 2. $\operatorname{dim} \Gamma=9$. Since there are no nine-dimensional quasi-simple Lie groups (see again [17]), the group $\Gamma$ in this case consists of at least two quasisimple factors. Since $\mathrm{K} \leq \mathrm{Spin}_{3} \mathbb{R}$, one of these factors must have a trivial maximal compact subgroup and hence must be isomorphic to the universal covering $\Omega$ of the group $S_{2} \mathbb{R}$. Because the center of $\Omega$ is infinite cyclic and is contained in the center $Z$ of $\Gamma$, we have $|Z|=\infty$. Choose an element $c \in \mathcal{T}$ such that $c^{Z} \neq c$. By $[4, X I .9 .3]$ and $[2,(3.3)]$ the ternary subfield $\mathcal{H}:=\left\langle c^{Z}\right\rangle$ is at least four-dimensional. Consequently, the stabilizer $\Gamma_{c}=\Gamma_{[\mathcal{H}]}$ is compact by $[14,2.3]$. Since $\operatorname{dim} \Gamma=9$ we have $\operatorname{dim} \Gamma_{c} \geq 1$, and thus $K \neq 1$. On the other hand, only one factor $\Phi$ of $\Gamma$ can have a non-trivial maximal subgroup. Thus we have the following subcases.

(a) $\Gamma=\Omega \Phi$. Then $\Phi \cong \mathrm{SL}_{2} \mathbb{C}$, for otherwise the group $\Gamma$ would contain commuting involutions and thus would be compact by $[14,2.3]$. Let $\omega$ denote the central involution of $\mathrm{SL}_{2} \mathbb{C}$. As before, we infer that $\Phi$ must fix the ternary subfield $\mathcal{F}_{\omega}$ pointwise, since $\Phi$ is a six-dimensional quasi-simple group. Again, this contradicts $[14,2.3]$, because the group $\mathrm{SL}_{2} \mathbb{C}$ is not compact.

(b) $\Gamma=\Omega_{1} \Omega_{2} \Phi$, where $\Omega_{1} \cong \Omega \cong \Omega_{2}$. Since the subgroup $\Phi$ contains a non-trivial maximal compact subgroup, there exists an involution $\omega$ in $\Phi$. Because the factors of $\Gamma$ commute pairwise, the involution $\omega$ possesses a centralizer $\Theta$ in $\Phi$ of dimension at least 7 , and the factors $\Omega_{1}$ and $\Omega_{2}$ are contained in $\Theta$. The centralizer $\Theta$ leaves the ternary subfield $\mathcal{F}_{\omega}$ invariant and the kernel of this action is compact. Thus, the six-dimensional group $\Omega_{1} \Omega_{2}$ must act effectively on $\mathcal{F}_{\omega}$ which contradicts the results of [1] and [2] or [15].

CASE 3. $\operatorname{dim} \Gamma=8$. As in Case 1 , the group $\Gamma$ is quasi-simple. Since $K \leq$ $\operatorname{Spin}_{3} \mathbb{R}$, the group $\Gamma$ is isomorphic to the universal covering of $S L_{3} \mathbb{R}$ or of $S U_{3,1} \mathbb{C}$, see [17]. But the group $\Gamma$ cannot be isomorphic to the universal covering of $\mathrm{SL}_{3} \mathbb{R}$, since this group contains a central involution $\omega$. As above this yields a contradiction, for $\Gamma$ would fix $\mathcal{F}_{\omega}$. So it remains to exclude the case that $\Gamma$ is isomorphic to the universal covering of $\mathrm{SU}_{3,1} \mathbb{C}$. For this, we shall apply Lemma (5). By Lemma (4) and Lemma (6) it suffices to verify condition(III) of Lemma (5). The centralizer $\Theta$ of an involution $\omega$ in $\Gamma$ is isomorphic to the universal covering of $U_{2} \mathbb{C}$. Consequently, this implies that $\operatorname{dim} \mathcal{M}=\operatorname{dim} \Gamma / \Theta=4$, because the group $U_{2} \mathbb{C}$ is four-dimensional. Hence, condition(III) is satisfied and by Lemma (5) the quotient $\Gamma / \Theta$ is compact. The group $\Gamma \cong \mathrm{SU}_{3,1} \mathbb{C}$ is simply connected and the centralizer $\Theta$ is connected. Thus the quotient $\Gamma / \Theta$ is also simply connected. This fact follows, for example, from the long exact sequence in homotopy which is induced by the short exact sequence $\mathbf{1} \rightarrow \Theta \rightarrow \Gamma \rightarrow \Gamma / \Theta \rightarrow \mathbf{1}$. An alternate proof can be found in $[11$, Ch. $2, \S 8$, Cor. 1]. Clearly, the natural action of $\Gamma$ on $\Gamma / \Theta$ is transitive. By $[10, \mathrm{Ch} . \mathrm{V}$, Th. (5.6)], a maximal compact subgroup of $\Gamma$ must also act transitively on $\Gamma / \Theta$, since this quotient is a simply connected compact manifold. But this is impossible, because a maximal compact subgroup of $\Gamma$ is three-dimensional and therefore cannot act transitively on a four-dimensional space. 
Altogether, we have shown that dim $\Gamma \leq 7$. Since no seven-dimensional semisimple Lie groups exist, the assertion of the theorem follows.

For the final estimation of the upper bound of $\operatorname{dim} \Gamma$ we need two more results which will be used later to ensure that we can apply Lemma (5).

(8) LEMMA. Let $\Gamma$ be a non-compact Lie group of dimension at least 8 and let $c \in \mathcal{V}$. Then the stabilizer $\Gamma_{c}$ is isomorphic to the group $\operatorname{Spin}_{3} \mathbb{R}$ or there exists an involution $\omega \in \Gamma$ such that $\operatorname{dim} \Gamma / C s(\omega)=4$.

Proof. Suppose that the stabilizer $\Gamma_{c}$ is not isomorphic to $\operatorname{Spin}_{3} \mathbb{R}$. Then we have $\Gamma_{c} \cong \mathbb{T}$ by Lemma (6). Moreover, the stabilizer $\Gamma_{c}$ is a maximal compact subgroup of $\Gamma$. Let $\omega$ be the unique involution of $\Gamma_{c}$ and set $\mathcal{M}:=\Gamma / \operatorname{Cs}(\omega)$. Suppose that $\operatorname{dim} \mathcal{M} \leq 3$. Then $\operatorname{dim} \operatorname{Cs}(\omega) \geq 5$ holds. Because the centralizer $\operatorname{Cs}(\omega)$ acts on the Baer ternary subfield $\mathcal{F}_{\omega}$ with a compact kernel and since the maximal compact subgroup of $\Gamma_{c}$ is a one-dimensional circle group, the centralizer $\operatorname{Cs}(\omega)$ induces on $\mathcal{F}_{\omega}$ a group of dimension at least 4 (note that $\Gamma_{c} \cong \mathbb{T}$ ), see [1, (3.2)]. But since $\mathcal{F}_{\Gamma}$ is connected, this contradicts $[15, \S 2,(9)]$.

(9) LEMMA. Let $\Gamma$ be a locally compact group containing an involution $\omega$. Let $\Phi$ and $\Psi$ be closed subgroups of $\Gamma$ such that $\Phi \unlhd \Psi$ and $\omega \in \operatorname{Cs}(\Phi) \cap \mathrm{N}_{\Gamma} \Psi$. If a maximal compact subgroup of $\Phi$ is trivial and if $\omega$ acts trivially on $\Psi / \Phi$, then the group $\Psi$ is contained in $\operatorname{Cs}(\omega)$.

Proof. Let $\psi \in \Psi \backslash \Phi$. Then $\psi \Phi=\psi^{\omega} \Phi$, i.e. there is some element $\varphi \in \Phi$ such that $\psi^{\omega}=\psi \varphi$. This implies that

$$
\psi=\psi^{\omega^{2}}=(\psi \varphi)^{\omega}=\psi^{\omega} \varphi^{\omega}=\psi^{\omega} \varphi=\psi \varphi^{2}
$$

and thus $\varphi^{2}=\mathbf{1}$. In particular, the element $\varphi$ lies in a maximal compact subgroup $\mathrm{K}$ of $\Phi$ (which exists because $\Phi$ is locally compact). But by assumption we have $\mathrm{K}=1$ and so $\varphi=1$. Hence $\psi^{\omega}=\psi$, i.e. $\psi \in \operatorname{Cs}(\omega)$.

(10) THEOREM. Let $\Gamma$ be a Lie group. If the ternary fixed field $\mathcal{F}_{\Gamma}$ is connected, then the group $\Gamma$ is compact or at most seven-dimensional.

Proof. Let $\Gamma$ be a non-compact Lie group. Suppose that $\operatorname{dim} \Gamma \geq 8$. Then $\Gamma$ is not semi-simple and a Levi complement $\Sigma$ of $\Gamma$ is of dimension at most 6 , see Theorem (7). To obtain a contradiction, we shall apply Lemma (5). By (4) and (6), we only have to verify condition (III) of Lemma (5). For this we may assume by Lemma (8) that a Levi complement $\Sigma$ contains a subgroup isomorphic to $\operatorname{Spin}_{3} \mathbb{R}$. Let $\mathrm{K}$ denote a maximal compact subgroup of $\Gamma$. Then the group $\mathrm{K}$ contains a subgroup isomorphic to $\operatorname{Spin}_{3} \mathbb{R}$, too. But, on the other hand, the group $K$ cannot contain commuting involutions by $[14,2.3]$ which implies that $K \cong \mathrm{Spin}_{3} \mathbb{R}$. Let $\omega$ denote the central involution of $\mathrm{K}$. The group $\mathrm{K}$ acts trivially on the ternary fixed field $\mathcal{F}_{\omega}$, else $\mathrm{K}$ would induce a non-trivial compact group on $\mathcal{F}_{\omega}$ by [5, (15)], and $\Gamma$ would thus be compact by $[14,2.3]$. Since $K$ is a closed quasi-simple subgroup of $\Gamma$, we may choose the Levi-Complement $\Sigma$ in such a way that $K$ is contained in $\Sigma$, see $[18,3.18 .13]$. Since $\Gamma$ is not semi-simple, there is a minimal connected 
commutative normal subgroup $\Xi$ in $\Gamma$. The subgroup $\Xi$ cannot be compact, since otherwise $\Xi$ would be a central subgroup of $K$ which is impossible because $K$ is semi-simple. Thus, the normal subgroup $\Xi$ is isomorphic to a vector group $\mathbb{R}^{t}$. Let $\Delta$ denote the solvable radical of $\Gamma$. By the theorem of Malcev-Iwasawa [7] the group $\Gamma$ is simply connected, because the maximal compact subgroup $K$ is simply connected. Consequently, by the Levi decomposition of simply connected groups, we have $\Sigma \cap \Delta=1$, see [18, Lemma 3.18.4]. By Theorem (7), the possible dimensions of $\Sigma$ are 3 and 6 . In the sequel we set $\Psi:=\operatorname{Cs}(\omega)$ and $\mathcal{M}:=\Gamma / \Psi$.

CASE $1 . \operatorname{dim} \Sigma=6$. Since the maximal compact subgroup $K$ of $\Sigma$ is isomorphic to $\operatorname{Spin}_{3} \mathbb{R}$, the group $\Sigma$ is isomorphic either to $\operatorname{Spin}_{3} \mathbb{C}$ or to a direct product of $\operatorname{Spin}_{3} \mathbb{R}$ and the universal covering $\Omega$ of $\mathrm{SL}_{2} \mathbb{R}$ (note that this product is direct, since the centers of the factors are $\mathbb{Z}_{2}$ and $\mathbb{Z}$ respectively and thus no identification of central elements can occur). In both cases the involution $\omega$ is central in $\Sigma$. Thus the group $\Sigma$ acts on $\mathcal{F}_{\omega}$. Since the compact kernel of this action contains $\mathrm{K}$, the group $\Sigma$ cannot be quasi-compact. In particular, the group $\Sigma$ cannot be isomorphic to $\operatorname{Spin}_{3} \mathbb{C}$. By $[15, \S 2,(9)],[5,(15)]$, and $[3,3.1]$ we conclude that $\operatorname{dim} \Psi \leq 6$, which implies that $\Psi^{1}=\Sigma$. In particular, the involution $\omega$ acts non-trivially on the normal subgroup $\Xi$. Since $\langle\omega\rangle$ is the center of the quasi-simple group $K$, this group must act effectively on $\Xi$. Because $\Gamma$ is at most ten-dimensional by $[3,3.5]$ and since $\operatorname{dim} \Sigma=6$, we have $\operatorname{dim} \Xi \leq 4$. But a faithful linear representation of $\operatorname{Spin}_{3} \mathbb{R}$ is at least four-dimensional. Hence, we conclude that dim $\Xi=4$. Therefore, $\operatorname{dim}\left(\Psi^{1} \ltimes \Xi\right)=\operatorname{dim} \Gamma$, and thus $\Gamma=\Psi^{1} \ltimes \Xi=\Psi \times \Xi$ holds, because $\Gamma$ is connected. Moreover, we have $\operatorname{dim} \mathcal{M}=4$, because $\Gamma / \Psi \approx \Xi$. So we can apply Lemma (5) which yields the compactness of $\mathcal{M}$. But this contradicts the fact that $\mathcal{M}=\Gamma / \Psi \approx \Xi \approx \mathbb{R}^{4}$. Consequently, the case $\operatorname{dim} \Sigma=6$ is impossible.

CASE $2 . \operatorname{dim} \Sigma=3$. In this case we have $\Sigma=K \cong \operatorname{Spin}_{3} \mathbb{R}$ and $\Delta \approx \mathbb{R}^{s}$. Let $n \in \mathbb{N}$ be such that $\Delta^{(n)} \neq \mathbb{1}$ but $\Delta^{(n+1)}=\mathbb{1}$. Then $\Delta^{(n)} \cong \mathbb{R}^{s_{n}}$ for some integer $s_{n}>0$. First, we shall show that $\operatorname{dim} \mathcal{M} \geq 4$ holds, so that we may apply Lemma (5) to conclude the compactness of $\mathcal{M}$. Then we shall verify that $\mathcal{M}$ is simply connected. This situation will give a contradiction similarly to the proof of Theorem (7).

Step 1. The quotient space $\mathcal{M}$ is a four-dimensional compact analytical manifold. Being a homogeneous space which is equivariantly homeomorphic to the quotient space of a Lie group modulo a closed subgroup, the set $\mathcal{M}$ is an analytical manifold. To prove the compactness of $\mathcal{M}$ we use Lemma (5). To apply this lemma, we only have to verify that $\operatorname{dim} \mathcal{M} \geq 4$ is satisfied. For this, choose $t \in \mathbb{N}$ minimal such that the involution $\omega$ does not act trivially on $\Upsilon:=\Delta^{(t-1)} / \Delta^{(t)} \cong \mathbb{R}^{s t}$. Such an integer $t$ exists, since else $\omega$ would act trivially on $\Delta$ (apply Lemma (9) $t$ times to $\Delta^{(l)}$ and $\Delta^{(l-1)}$, where $\left.1 \leq l \leq t\right)$. This would imply that $\Delta \leq \Psi$ and $\omega$ would be a central involution of $\Gamma$. Consequently, we would have $\operatorname{dim} \Gamma \leq 6$ which would be a contradiction to our assumption $\operatorname{dim} \Gamma \geq 8$. Hence, the involution $\omega$ 
and thus the group $\Sigma$ acts non-trivially on the quotient $\Upsilon$. Because of the minimality of $t$ we have $\Delta^{(t)} \leq \Psi$ by (9). The involution $\omega$ splits $\Upsilon$ into the direct sum $\Upsilon=\Upsilon_{+} \oplus \Upsilon_{-}$of the eigenspaces corresponding to the eigenvalues 1 and -1. The eigenspaces $\Upsilon_{+}$and $\Upsilon_{-}$are $\Gamma$-invariant, because $\omega$ is central in $\Sigma$. Let $\Pi:=\bigcup \Upsilon_{+} \leq \Delta^{(t-1)}$ be the preimage of $\Upsilon_{+}$with respect to the canonical projection $\Delta^{(t-1)} \rightarrow \Upsilon$. The involution $\omega \in \Sigma \cong \operatorname{Spin}_{3} \mathbb{R}$ acts non-trivially on $\Delta^{(t-1)} / \Pi$ (use again Lemma (9)). This implies that $\Sigma$ acts effectively on $\Delta^{(t-1)} / \Pi \cong \mathbb{R}^{k}$. Hence we have $k \geq 4$, since the group $\operatorname{Spin}_{3} \mathbb{R}$ has no faithful representation of dimension less than 4 . The involution $\omega$ acts trivially on $\Pi$ by definition of $\Pi$ which implies that $\Pi \leq \Psi \cap \Delta^{(t-1)}$. On the other hand, if $\delta \in \Psi \cap \Delta^{(t-1)}$ then $\delta^{\omega}=\delta$ which implies that $\delta \in \Pi$. Hence we have $\Pi=\Psi \cap \Delta^{(t-1)}$. The groups $\Delta^{(t-1)} \Psi / \Psi$ and $\Delta^{(t-1)} /\left(\Psi \cap \Delta^{(t-1)}\right)=\Delta^{(t-1)} / \Pi$ are isomorphic as topological groups, see [6, Th. 5.33]. In particular, we have

$$
\operatorname{dim} \mathcal{M}=\operatorname{dim} \frac{\Gamma}{\Psi} \geq \operatorname{dim} \frac{\Delta^{(t-1)} \Psi}{\Psi}=\operatorname{dim} \frac{\Delta^{(t-1)}}{\Pi}=k \geq 4 .
$$

Thus, we may apply Lemma (5) to conclude that $\mathcal{M}$ is compact and four-dimensional. Furthermore, this implies that in (1) actually we have equality throughout.

Step 2 . The manifold $\mathcal{M}$ is simply connected. According to the representation $\Gamma=\Sigma \times \Delta$ there exists a subgroup $\Delta_{\Psi} \leq \Delta$ such that $\Psi=\Sigma \ltimes \Delta_{\Psi}$. By $[11$, Ch. $2, \S 8$, Cor. 1$]$, the quotient $\mathcal{M}=\Gamma / \Psi$ is simply connected if and only if $\Psi$ is connected. Since $\Sigma$ is connected and $\Psi$ can be written as $\Psi=\Sigma \ltimes \Delta_{\Psi}$ it suffices to verify the connectedness of $\Delta_{\Psi}$.

Using the notation of Step 1 we have $\Delta^{(t-1)} \cap \Delta_{\Psi} \leq \Pi$ and thus $\Delta^{(t-1)} \cap \Delta_{\Psi}=$ $\Pi \cap \Delta_{\Psi}$. Setting $\Delta_{\Psi}^{(i)}:=\Delta^{(i)} \cap \Delta_{\Psi}$, we obtain

$$
\operatorname{dim} \Delta_{\Psi}=\left(\sum_{i \neq t} \operatorname{dim} \frac{\Delta_{\Psi}^{(i-1)}}{\Delta_{\Psi}^{(i)}}\right)+\operatorname{dim} \frac{\left(\Pi \cap \Delta_{\Psi}\right)}{\Delta_{\Psi}^{(t)}} .
$$

On the other hand, by relation (1) we have $\operatorname{dim} \mathcal{M}=\operatorname{dim} \Delta^{(t-1)} / \Pi=4$ and thus

$$
\left(\sum_{i \neq t} \operatorname{dim} \frac{\Delta^{(i-1)}}{\Delta^{(i)}}\right)+\operatorname{dim} \frac{\Pi}{\Delta^{(t)}}=\operatorname{dim} \Delta-4
$$

holds. Considering the representations $\Gamma=\Sigma \ltimes \Delta$ and $\Psi=\Sigma \ltimes \Delta_{\Psi}$ we infer that $\mathcal{M}=\Gamma / \Psi \approx \Delta / \Delta_{\Psi}$. Consequently, by [12, Th. 2.1$]$ we have the equation

$$
\operatorname{dim} \Delta_{\Psi}=\operatorname{dim} \Delta-4 \text {. }
$$

Since $\Delta_{\Psi}^{(i)}=\Delta^{(i)} \cap \Delta_{\Psi}=\Delta^{(i)} \cap \Delta_{\Psi}^{(i-1)}$ this implies that

$$
\frac{\Delta_{\Psi}^{(i-1)}}{\Delta_{\Psi}^{(i)}}=\frac{\Delta_{\Psi}^{(i-1)}}{\left(\Delta^{(i)} \cap \Delta_{\Psi}^{(i-1)}\right)} \cong \frac{\Delta^{(i)} \Delta_{\Psi}^{(i-1)}}{\Delta^{(i)}} \leq \frac{\Delta^{(i-1)}}{\Delta^{(i)}} .
$$


Similarly, from the relation $\Delta_{\Psi}^{(t)}=\Delta^{(t)}$ (use $\Delta^{(t)} \leq \Psi$ ) we infer that

$$
\frac{\left(\Pi \cap \Delta_{\Psi}\right)}{\Delta_{\Psi}^{(t)}}=\frac{\left(\Pi \cap \Delta_{\Psi}\right)}{\Delta^{(t)}} \leq \frac{\Pi}{\Delta^{(t)}} .
$$

Combination of the relations (2) to (6) yields the equations

$$
\operatorname{dim} \frac{\Delta_{\Psi}^{(i-1)}}{\Delta_{\Psi}^{(i)}}=\operatorname{dim} \frac{\Delta^{(i-1)}}{\Delta^{(i)}} \quad(i \neq t)
$$

and

$$
\operatorname{dim} \frac{\left(\Pi \cap \Delta_{\Psi}\right)}{\Delta_{\Psi}^{(t)}}=\operatorname{dim} \frac{\Pi}{\Delta^{(t)}}
$$

for $1 \leq i \leq n+1$. Consequently, by relations (5) and (6), we obtain the following isomorphisms between topological groups

$$
\begin{aligned}
& \frac{\Delta_{\Psi}^{(i-1)}}{\Delta_{\Psi}^{(i)}} \cong \frac{\Delta^{(i-1)}}{\Delta^{(i)}} \cong \mathbb{R}^{s_{i}} \quad(i \neq t) \\
& \frac{\Delta_{\Psi}^{(t-1)}}{\Delta_{\Psi}^{(t)}}=\frac{\left(\Delta^{(t-1)} \cap \Delta_{\Psi}\right)}{\Delta_{\Psi}^{(t)}}=\frac{\left(\Pi \cap \Delta_{\Psi}\right)}{\Delta_{\Psi}^{(t)}} \cong \frac{\Pi}{\Delta^{(t)}} \cong \mathbb{R}^{s_{t}} .
\end{aligned}
$$

Thus the connectedness of $\Delta_{\Psi}$ can be verified inductively from the following part of the long exact homotopy sequence

$$
\pi_{1}\left(\frac{\Delta_{\Psi}^{(i-1)}}{\Delta_{\Psi}^{(i)}}\right) \rightarrow \pi_{0}\left(\Delta_{\Psi}^{(i)}\right) \rightarrow \pi_{0}\left(\Delta_{\Psi}^{(i-1)}\right) \rightarrow \pi_{0}\left(\frac{\Delta_{\Psi}^{(i-1)}}{\Delta_{\Psi}^{(i)}}\right) .
$$

For this, we first note that by the isomorphisms (7) and (8) we have

$$
\pi_{1}\left(\frac{\Delta_{\Psi}^{(i-1)}}{\Delta_{\Psi}^{(i)}}\right)=\mathbb{1}=\pi_{0}\left(\frac{\Delta_{\Psi}^{(i-1)}}{\Delta_{\Psi}^{(i)}}\right)
$$

and thus the sequence above reduce to

$$
\mathbf{1} \rightarrow \pi_{0}\left(\Delta_{\Psi}^{(i)}\right) \rightarrow \pi_{0}\left(\Delta_{\Psi}^{(i-1)}\right) \rightarrow \mathbf{1} .
$$

Hence, via induction the sets $\pi_{0}\left(\Delta_{\Psi}^{(i)}\right)$ have equal cardinality for all $0 \leq i \leq n+1$. But since $\Delta_{\Psi}^{(n+1)} \leq \Delta^{(n+1)}=1$ is connected, this implies that all groups $\Delta_{\Psi}^{(i)}$ and in particular $\Delta_{\Psi}=\Delta_{\Psi}^{(0)}$ is connected which proves the assertion of Step 2. 
Step 3. The contradiction. Since $\Gamma$ acts transitively on the simply connected compact manifold $\mathcal{M}$, the maximal compact subgroup $\mathrm{K}$ of $\Gamma$ acts transitively, too, on $\mathcal{M}$, see $[10, \mathrm{Ch}$. V, Th. (5.6)]. But as we have shown before, this is a contradiction to $\operatorname{dim} \mathcal{M}=4$ and $\operatorname{dim} K=3$. Thus the assumption that the dimension of $\Gamma$ is at least 8 is contradictory and the theorem is proved.

Summing up, we get the following result:

(11) COROLLARY. If $\Gamma$ is a Lie group having a connected ternary fixed field $\mathcal{F}_{\Gamma}$, then either $\Gamma$ is isomorphic to one of the groups $G_{2(-14)}$ or $\mathrm{SU}_{3} \mathbb{C}$, or the dimension of $\Gamma$ is at most 7.

\section{References}

1. Bödi, R.: On the dimensions of automorphism groups of 4-dimensional double loops, Math. $Z$. 215 (1994), 89-97.

2. Bödi, R.: On the dimensions of automorphism groups of 8-dimensional double loops, Monatsh. Math. 117 (1994), 1-16.

3. Bödi, R.: On the dimensions of automorphism groups of 8-dimensional ternary fields, I (to appear in $J$. Geometry).

4. Grundhöfer, T. and Salzmann, H.: Locally compact double loops and ternary fields, in Quasigroups and Loops: Theory and Applications, O. Chein, H. D. Pflugfelder, J. D. H. Smith, (eds), Heldermann, Berlin, 1990.

5. Grundhöfer, T. and Stroppel, M.: On restrictions of automorphism groups of compact projective planes to subplanes, Results Math. 21 (1992), 319-327.

6. Hewitt, E. and Ross, K. A.: Abstract Harmonic Analysis, I, 2nd edn, Springer, Berlin, Heidelberg, New York, 1979.

7. Iwasawa, K.: On some types of topological groups, Ann. Math. 50 (1949), 507-558.

8. Kelley, J. L.: General Topology, van Nostrand, Princeton, N.J., 1955.

9. Löwen, R.: Topology and dimension of stable planes: On a conjecture of H. Freudenthal, J. reine angew. Math. 343 (1983), 108-122.

10. Montgomery, D. and Zippin, L.: Topological Transformation Groups, Wiley, New York, 1955.

11. Mostow, G. D.: The extensibility of local Lie groups of transformations, Ann. Math. 52 (1950), 606-636.

12. Nagami, K.: Dimension-theoretical structure of locally compact groups, J. Math. Soc. Japan 14 (1962), 379-396.

13. Pears, A. R.: Dimension Theory of General Spaces, Cambridge University Press, 1975.

14. Salzmann, H.: Automorphismengruppen achtdimensionaler Ternärkörper, Math. Z. 166 (1979), 265-275.

15. Salzmann, H.: Compact 8-dimensional projective planes with large collineation groups, Geom. Dedicata 8 (1979), 139-161.

16. Salzmann, H.: Topological planes, Adv. Math. 2 (1967), 1-60.

17. Tits, J.: Tabellen zu den einfachen Liegruppen und ihren Darstellungen, Lecture Notes in Math. 40, Springer-Verlag, Berlin, Heidelberg, New York, 1967.

18. Varadarajan, V. S.: Lie Groups, Lie Algebras, and Their Representations, Springer, Berlin, Heidelberg, New York, 1984. 